\title{
Some active processes in comet icy nuclei: nucleus splitting and anti tail formation
}

\author{
Kh. I. Ibadinov, A. M. Buriev, and A. G. Safarov \\ Institute of Astrophysics, Academy of Sciences of Tajikistan, Tajikistan \\ email: Ibadinov@mail.ru
}

\begin{abstract}
The most dramatic display of variable activity of a comet is splitting of the nucleus. For the purpose of revealing the trends of splitting of comet nuclei and of formation of abnormal cometary tails, we have created two catalogues of comets: a catalogue of split nuclei, containing 99 comets, and a catalogue of comets with abnormal tails, including 60 objects. Statistical investigation reveals some general trends of these phenomena. The greatest number of recorded cases of nucleus splitting and abnormal tail (60\%) occurs within an interval of heliocentric distance ranging from 0.6 $\mathrm{AU}$ to $1.6 \mathrm{AU}$ (maximum at $1.1 \mathrm{AU}$ ) and geocentric distance ranging from 0.6 AU to $1.8 \mathrm{AU}$ (maximum at $1.15 \mathrm{AU}$ ). Splitting of nuclei and abnormal tails are more often $(75 \%)$ recorded close to the perihelia of the cometary orbits. Only $16 \%$ of splitting comets also exhibit abnormal tails. Some cases of nuclear splitting and large velocity (some $\mathrm{km} / \mathrm{s}$ ) eruptions of dust from a nucleus, as well as cases of abnormal tails developed at large heliocentric distances, may indicate collisions of comet nuclei with other bodies. Our results are of interest for the physics of comets, and for the distribution of meteoroids in solar system.
\end{abstract}

Keywords. Comets, Comet: nucleus, Come: splitting

\section{Introduction}

Comets are extremely variable objects, exhibiting brightness flashes, gas-dust jets, halos, heterogeneity in plasma tailis, abnormal tails and splitting of the nucleus. The most dramatic display of variable activity of a comet is nucleus splitting. As a result of splitting, observable daughter comets and meteoroid stream are formed; sometimes the comet disappears completely. Another kind of unusual activity of comet nuclei is the abnormal direction of the tail: towards the Sun rather than in the antisolar direction. It was suggested that such abnormal tails result from synchronous emission of meteoroid particles from the nucleus towards the Sun (Bredikhin 1934; Vsekhsvytskiy 1958). Splitting of the nucleus and formation of an abnormal tail attest to active processes that may take place in cometary nuclei. It is possible that both phenomena are the consequence of one process. The reasons and mechanisms of splitting of the nucleus and anti-tail formation are not always known. Very often it is not possible to define the exact time of onset of these phenomena.

The purpose of the present work is to reveal from observations the general trends of nucleus splitting and abnormal tail formation. To achieve this purpose, we we have created two catalogues. The first includes 99 comets for which nuclear splitting, or obvious signs of splitting have been recorded; it is a continuation of similar catalogues of Konopleva (1967), Golubev (1975), Dobrovolsky \& Gerasimenko (1987) and Ibadinov (1998). The second catalogue includes 60 comets for which an abnormal tail was observed and provides a considerable supplement to Demenko's (1965) catalogue, which included only 16 comets. These catalogues will be published in a separate paper. On the basis of these catalogues, we investigate the conditions of nucleus splitting and formation of an 
abnormal tail in comets. We consider the dependence of these phenomena on the heliocentric $(r)$ and geocentric $(\Delta)$ distances of the comet at the moment of their detection, on the inclination of the orbital plane of the comet with respect to the ecliptic $(i)$, and on the perihelion distance $(q)$ of the orbit. In the present paper we focus on statistical results, presenting the number of splitting comets $N$ and the number of comets with an abnormal tail $N_{t}$ as functions of each of the parameters $r, \Delta, i$ and $q$.

In Fig.1 (a-b-c) histograms are presented, showing the dependence of the number of splitting comets $N$ on heliocentric distance $r$ and geocentric distance $\Delta$ at the moment of registration of the splitting and the dependence of $N$ on perihelion distance of the comet's orbit. Similar histograms are presented in Fig. $2(\mathrm{a}-\mathrm{b}-\mathrm{c})$ for comets with abnormal tails. It is noticeable that the maximum number of splittings and abnormal tails occurs within an interval of heliocentric and geocentric distances between 0.6-1.6 AU, which most likely reflects the visibility conditions of comets, i.e., near the orbit of the Earth, nucleus splitting and abnormal tails are more easily observed than far from it. The same effect is exhibited in Fig. 1(a) and 2(c), showing the dependence of $N$ and $N_{t}$ on $q$, where maximum is obtained again in the range 0.6-1.6 AU. In our opinion, this effect also is caused by observational bias (near Earth's orbit).

By contrast, the results presented in Fig. 1(d) and Fig. 2(d) can shed light on the general trends and the most probable mechanisms responsible for splitting of comet nuclei and formation of abnormal tails. In Fig. 1(d) the dependence of the number of comets $N$ on the difference between the heliocentric distance $r$ where splitting was recorded and perihelion distance $q$, i.e. $(r-q)$, is presented. We note that the maximum is obtained near $(r-q) \approx 0$. This result confirms Dobrovolsky \& Gerasimenko (1987) and Ibadinov (1998) conclusions, that the maximum number of splitting of comets occurs near perihelion. We obtain similar results for the abnormal tails of comets: in Fig. 2(d) the dependence of the number of comets with an abnormal tail on $(r-q)$ is presented and the maximum
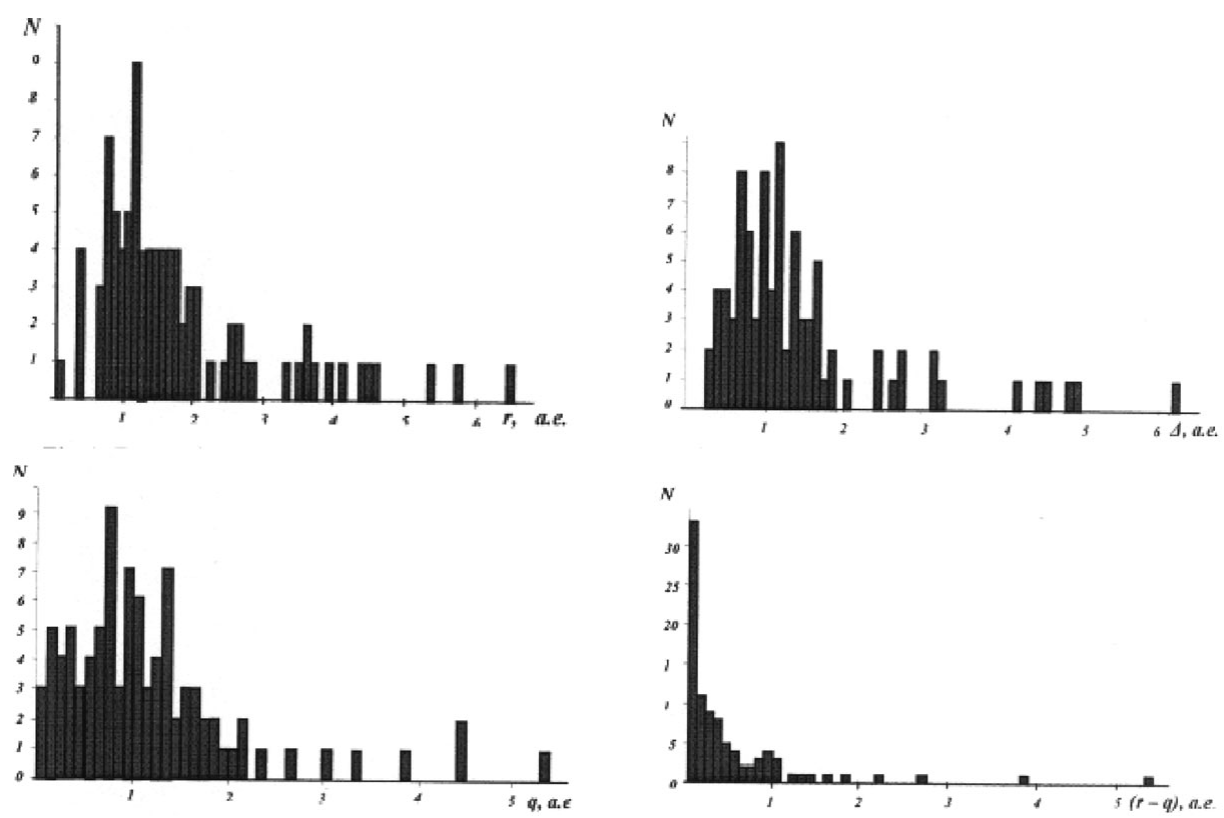

Figure 1. Splitting nuclei of comets. Dependence of number of comets $N$ on: (a) heliocentric distance $r$; (b) geocentric distance $\Delta$; (d) perihelion distance $q$; (e) $(r-q)$. 
of the distribution $N(r-q)$ occurs again near $(r-q) \approx 0$, that is, in the vicinity of the perihelion.

In the distribution $N(r, \Delta, q,(r-q))$ separate peaks at large distances are observed, in the interval 3-5 AU. Here, the influence of Jupiter is considerable. This group of comets includes comet Hailey-Bopp, which has collapsed under the influence of Jupiter. It may be also possible that in this region collisions of comets with asteroids and meteoroids take place as well.

In summary, from the statistical analysis of comet nucleus splitting it is established with sufficient confidence that the highest probability for splitting is obtained near orbital perihelion. The probability of detecting nucleius splitting is highest when the comet is within heliocentric and geocentric distances 0.6-1.6 AU, due to observational bias (best observing conditions).
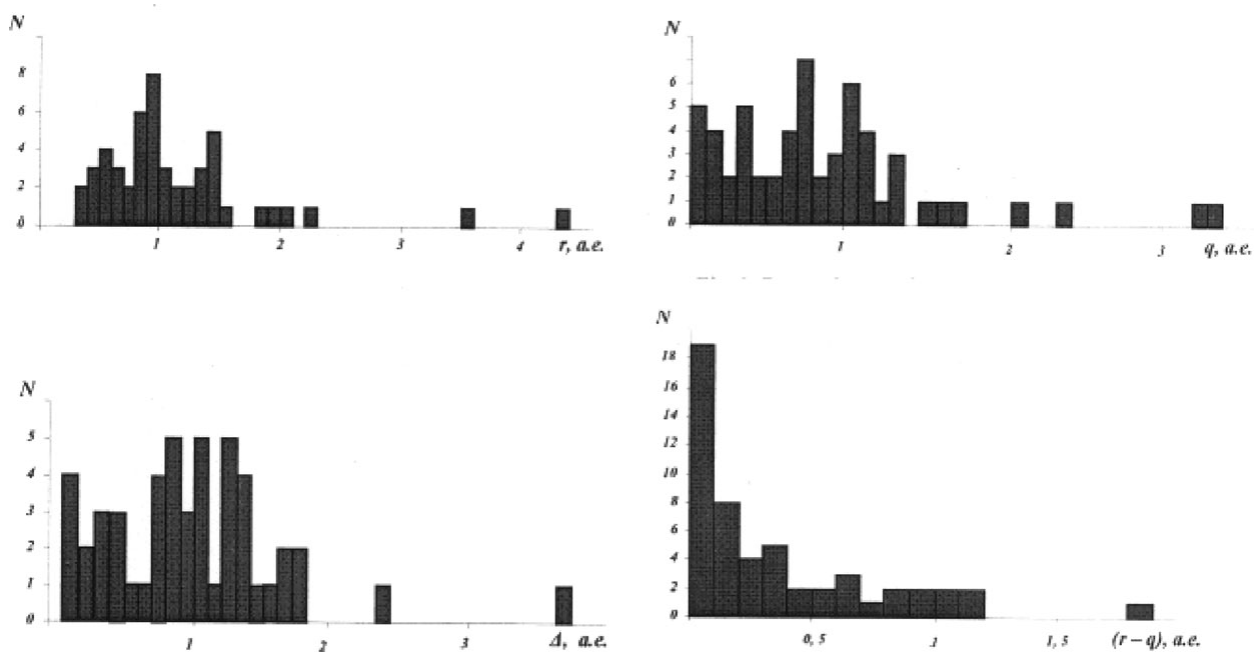

Figure 2. Anti-tail of comets. Dependence of number of comets $N$ with anti-tail on: (a) heliocentric distance $r$; (b) perihelion distance $q$; (d) geocentric distance $\Delta$; (e) $(r-q)$.

\section{Conclusions}

We have created catalogues of recorded comet nucleus splittings and abnormal cometary tails. Based on statistics, it is established that the probability of detecting the splitting of comet nuclei and the formation of abnormal tails is high, if these phenomena have occurred within heliocentric and geocentric distances in the range 0.6-1.6 AU. This is probably connected with observing conditions. The greatest number of splittings and abnormal tails is recorded near the perihelia of the cometary orbits. A plausible reason for splitting may be the tidal influence of the Sun and of Jupiter. We find that abnormal cometary tails are also formed at large distances from the Sun, between the orbits of Mars and Jupiter. Only for $16 \%$ of splitting comets were abnormal tails also detected.

\section{References}

Bredikhin, F. A. 1934, About tails of comets, Rus., M: GTTI

Golemubev, V. A. 1975, Problems of Space Physics, 10, 23 
Demenko, A. A. 1965, About tails of comets, (dissertation), Kiev

Dobrovolsky, O. V., \& Gerasimenko, S. I. 1987, Bull. of Institute of Astrophysics of AS RT 77,3

Ibadinov, K. I. 1998, Space Research Institute of Russian Academy of Sciences, p. 296

Konopleva, V. P. 1967, in: Active processes in comets, (Kiev: Naukova Dumka), p. 57

Vsekhsvytskiy, S. K. 1958, Physical properties of comets, M. Prosveshenie 